\title{
Primary mediastinal giant liposarcoma with smooth muscle and neural differentiation: A case report
}

\author{
JIAN MA $^{1 *}$, HONG-MEI ZHANG ${ }^{2 *}$, LI-WANG ZHANG ${ }^{3}$, MIN-WEN ZHENG ${ }^{4}$ and MIN YU ${ }^{5}$ \\ ${ }^{1}$ Department of Research, Tangdu Hospital, The Fourth Military Medical University, Xi'an, Shaanxi 710038; \\ ${ }^{2}$ Department of Oncology, Xijing Hospital, The Fourth Military Medical University, Xi'an, Shaanxi 710032; \\ ${ }^{3}$ Department of General Surgery, Tangdu Hospital, The Fourth Military Medical University, Xi'an, Shaanxi 710038; \\ ${ }^{4}$ Department of Radiology, Xijing Hospital; ${ }^{5}$ Department of Military Health Services, \\ The Fourth Military Medical University, Xi'an, Shaanxi 710032, P.R. China
}

Received March 23, 2014; Accepted December 12, 2014

DOI: $10.3892 / \mathrm{ol} .2015 .3103$

\begin{abstract}
Liposarcoma has previously been described in Western studies, however, such cases are rarely reported in the mediastinum. In addition, the presence of a liposarcoma with smooth muscle and neural differentiation has not been previously reported. Thus, the present study describes the rare case of a 28-year-old Chinese male admitted to our hospital with the symptoms of chest tightness and shortness of breath due to a recurrent fibrolipoma in the mediastinum. The resected tumor measured $23 \mathrm{~cm}$ at its largest diameter, with histopathological and immunohistochemical features indicating a well-differentiated liposarcoma accompanied by smooth muscle and neural differentiation. Following the resection, the patient underwent radiation treatment and remains alive with no evidence of disease recurrence at two months post-surgery. To the best of our knowledge, the present study is the first to report a case of liposarcoma with smooth muscle and neural differentiation, which indicates that liposarcomas could potentially originate from stem cells. The present study highlights the fact that pathologists must carefully investigate the histopathological characteristics of liposarcomas in order to obtain an accurate diagnosis.
\end{abstract}

Correspondence to: Professor Min-Wen Zheng, Department of Radiology, Xijing Hospital, The Fourth Military Medical University, 15 Changle xi Road, Xi'an, Shaanxi 710032, P.R. China

E-mail: zhengminwenfmmu@163.com

Professor Min Yu, Department of Military Health Services, The Fourth Military Medical University, 169 Changle xi Road, Xi'an, Shaanxi 710032, P.R. China

E-mail:kfyxkfg@fmmu.edu.cn

*Contributed equally

Key words: liposarcoma, mediastinum, differentiation, smooth muscle, neural

\section{Introduction}

Liposarcoma is the most common type of soft-tissue sarcoma, typically occurring in the lower extremities and retroperitoneum and accounts for 10-16\% of all sarcomas (1). Its diagnosis depends mainly on histopathological characteristics such as typical lipoblasts, and immunohistochemical features, namely MDM2, CDK4 and S-100 were positive (2). By contrast, mediastinal liposarcomas are rare and constitute $<1 \%$ of all mediastinal tumors worldwide (3). Few cases have been described in the English literature (4-6). Furthermore, the majority of cases were of giant liposarcomas $(5,6)$. In addition, Boland et al (4) considered that the majority of patients with well-differentiated/undifferentiated liposarcoma had a protracted clinical progression, in contrast to the more rapid disease progression observed in patients with myxoid and plemorphic liposarcoma. Liposarcomas originate from primitive mesenchymal cells rather than mature fat cells (7). Thus, it may differentiate towards various mesenchymal tissues. The current study presents a rare case of a giant liposarcoma with smooth muscle and neural differentiation in the mediastinum. Written informed consent was obtained from the family of the patient.

\section{Case report}

A 27-year-old male was referred to the Thoracic Surgical Hospital, (Weinan, China) in June 2012 due to a one-month history of chest tightness and shortness of breath at rest. Chest computed tomography revealed a bulky, well-defined mass in the posterior mediastinum, which was subsequently resected by thoracotomy and diagnosed as a fibrolipoma. Following the discharge of the patient, no therapy was received. The patient was admitted to Tangdu Hospital (Xi'an, China) in September 2013 with the complaint of increasing chest tightness and shortness of breath 12 months after the initial thoracotomy had been performed. Imaging features revealed a large mass extending from the posterior mediastinum to the neck, indicating tumor recurrence. The patient consequently underwent surgery to excise the mass. During the surgical procedure the tumor was identified to have adhered to the 

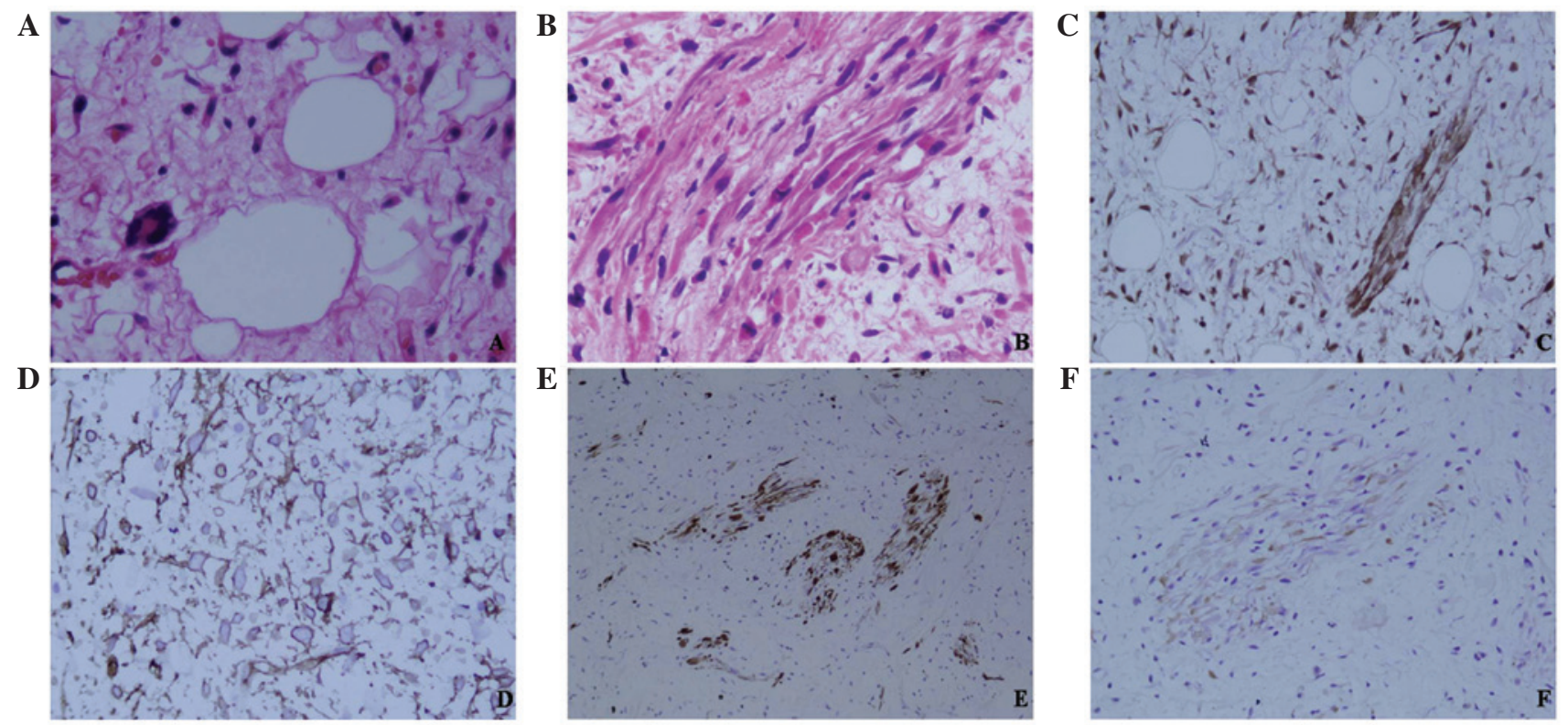

Figure 1. Microscopically, the tumor was composed of (A) fat cells and (B) spindle cells organized in a fascicular pattern (magnification, x400; hematoxylin and eosin staining). Immunohistochemically, the spindle cells were positive for (C) cyclin-dependent kinase-4 (magnification, x100), (D) cluster of differentiation 34 (magnification, x200), (E) desmin (magnification, x100) and (F) neurofilament (magnification, x100).

lower thyroid gland, compressing the superior vena cava and the right wall of the trachea. Following complete excision of the mass, the tumor was found to be $23 \times 15 \times 10 \mathrm{~cm}$ in size, with a yellow cut surface. Its circumsciption was clear, with no conglutination with the surrounding tissues, furthermore, the texture was soft. Microscopically, the tumor was composed of spindle cells organized in a fascicular pattern and fat cells (Fig. 1A and B). Lipoblast cells were identified, however, they were not abundant, and mitotic figures were absent. Immunohistochemically, the spindle cells were positive for vimentin, cyclin-dependent kinase (CDK)-4 (Fig. 1C), cluster of differentiation (CD) 34 (Fig. 1D), desmin (Fig. 1E), smooth muscle actin (SMA), myogenin and neurofilament (NF; Fig. 1F), and negative for glial fibrillary acidic protein, myoglobin, S-100, epithelial membrane antigen, leukocyte common antigen, B-cell lymphoma-2, CD99 and CD117. Thus, the tumor was diagnosed as a well-differentiated liposarcoma with smooth muscle and neural differentiation. A histological comparison between the initial tumor slides obtained from the local hospital and the reoccurring tumor tissue demonstrated evident differences. The initial tumor was composed of fiber and fat tissues and no lipoblast cells were identified; thus, there was no evidence of a well-differentiated liposarcoma. Following surgery, the patient underwent radiation treatment, a dose of 2 Gy was given five times a week, for 4-5 weeks.. The patient remains alive with no evidence of disease recurrence at two months post-surgery.

\section{Discussion}

Liposarcoma arises from primitive mesenchymal cells and commonly occurs in the lower extremities (75\% of cases), with a less frequent rate of occurrence in the retroperitoneum (20\% of cases) (8-10). Alternative sites of liposarcoma, such as the abdomen, vulva and buttocks, have also been reported (11); however, primary liposarcoma of the mediastinum is rare, comprising $<1 \%$ of all mediastinal tumor cases (only 130 cases have been described in the Western literature thus far) (3). The majority of cases occur in patients aged $>40$ years, with a mean age range of 43-58 years (total age range, 3-72 years). Furthermore, there is no apparent difference in the incidence rate between genders $(12,13)$. Mediastinal liposarcomas may grow to a large size (mean tumor size, $15.7 \mathrm{~cm}$; range, $2.2-61 \mathrm{~cm}$ ), compressing the surrounding organs and causing symptoms such as shortness of breath, chest pain, dyspnea and tachypnea $(14,15)$. The patient in the present study was young (age, 27 years) and exhibited a tumor with a large diameter $(23 \mathrm{~cm})$, which caused it to compress the trachea.

Liposarcoma is histologically classified into the following five subtypes, according to the most recent World Health Organization Classification of Soft Tissue Tumors: i) Well-differentiated, ii) myxoid, iii) round cell, iv) dedifferentiated and v) pleomorphic (2). These histological subtypes correlate with the clinical behavior and prognosis; for example, the excessive size of mediastinal liposarcomas may result in invasion of the surrounding structures, such as the pericardium, superior vena cava, heart, trachea, bronchi and esophagus, causing symptoms such as shortness of breath, dyspnea and pain. Additionally, the prognosis of patients with dedifferentiated or pleomorphic liposarcomas is poor compared with patients with well-differentiated liposarcomas (12). Despite its rapid recurrence, the case presented in the current study may be histologically classified as a well-differentiated liposarcoma, according to its histological features. However, its immunohistochemical features, including spindle cells positive for CD34, CDK4, NF, desmin and SMA, indicate a diagnosis of well-differentiated liposarcoma with smooth muscle and neural differentiation. To the best of our knowledge, the present study is the first to report this specific subtype of liposarcoma.

The recurrence of established liposarcoma is common, with the majority of cases reoccurring within the first six months, although recurrence may be delayed for up to 
5-10 years following the initial excision (16). In the present case, the patient exhibited tumor recurrence 12 months after discharge from the local hospital. Despite an initial diagnosis of a benign fibrolipoma, the second resected tumor indicated a low-grade malignant liposarcoma. One possible explanation of these different diagnoses is that the primitive tumor became malignant one year after the surgery. An alternative explanation is that the true malignant lesion was not identified as only a limited sample was resected. Overall, the present study indicates a requirement for long-term and careful follow-up for tumors in the mediastinum, regardless of whether the tumor was initially diagnosed as benign.

Surgical removal with a wide margin is the optimal treatment strategy for mediastinal liposarcoma, with chemotherapy and radiotherapy appearing ineffective as adjuncts to surgical excision in unresectable or incompletely resected tumors $(17,18)$. The prognosis is dependent on the histological subtype and the completion of surgical resection, with myxoid or pleomorphic subtype patients exhibiting a poorer prognosis compared with well-differentiated tumors (12). Furthermore, a number of previous studies have reported a five-year survival rate of $34.9 \%$ for the well-differentiated subtype and $21.0 \%$ for the pleomorphic subtype $(8,10,19)$.

In conclusion, to the best of our knowledge, the current study is the first to report a case of well-differentiated liposarcoma with smooth muscle and neural differentiation in the mediastinum. Furthermore, the study indicates the requirement for a long-term follow-up period for patients with mediastinal tumors, even after a diagnosis of a benign tumor arising from the mesenchymal tissue. Multiple tissues should be sampled and histologically investigated to achieve an accurate diagnosis.

\section{References}

1. Conesa X, Seijas R, Ares O, et al: Multicentric liposarcoma. Acta Orthop Belg 77: 9-14, 2011.

2. Fletcher CDM, Unni KK, Mertens F, (eds.): Pathology and genetics of tumours of soft tissue and bone. In: World Health Organization Classification of Tumours. IARC Press, Lyon, France, pp35-44, 2002.
3. Kashu Y, Yukumi S, Tsunooka N, Tanigawa K, Arakane M, Nakagawa $\mathrm{H}$ and Kawachi K: Successful resection of a massive mediastinal liposarcoma that rapidly extended into the entire left thoracic cavity: report of a case. Surg Today 42: 68-71, 2012.

4. Boland JM, Colby TV and Folpe AL: Liposarcomas of the mediastinum and thorax: a clinicopathologic and molecular cytogenetic study of 24 cases, emphasizing unusual and diverse histologic features. Am J Surg Pathol 36: 1395-1403, 2012.

5. Matsuo T, Takamori S, Hayabuchi N, et al: Giant liposarcoma occupying most of the hemi-thorax and resected in the supine position: report of a rare case. Kurume Med J 58: 63-65, 2011.

6. Németh T, Géczi T, Tiszlavicz L, Wolfárd A, Lázár G and Furák J: Giant fibrolipoma of the mediastinum. Magy Seb 64: 235-238, 2011 (In Hungarian).

7. Sung MS, Kang HS, Suh JS, et al: Myxoid liposarcoma: appearance at MR imaging with histologic correlation. Radiographics 20: 1007-1019, 2000

8. Ohta Y, Murata T, Tamura M, Sato H, Kurumaya $\mathrm{H}$ and Katayanagi K: Surgical resection of recurrent bilateral mediastinal liposarcoma through the clamshell approach. Ann Thorac Surg 77: 1837-1839, 2004.

9. Caraglia M, Montella L, Addeo R, et al: Conditions suggesting lymphoma: case 2. Mediastinal liposarcoma in a patient with previous testicular cancer. J Clin Oncol 23: 3844-3846, 2005.

10. Noji T, Morikawa T, Kaji M, Ohtake S and Katoh H: Successful resection of a recurrent mediastinal liposarcoma invading the pericardium: report of a case. Surg Today 34: 450-452, 2004.

11. Raghavan R, Raghuram P, Parekh PV and Kurien JM: Posterior mediastinal liposarcoma simulating a lung mass: an unusual case report. Cancer Imaging 7: 141-144, 2007.

12. Klimstra DS, Moran CA, Perino G, Koss MN and Rosai J: Liposarcoma of the anterior mediastinum and thymus. A clinicopathologic study of 28 cases. Am J Surg Pathol 19: 782-791, 1995.

13. Hahn HP and Fletcher CD: Primary mediastinal liposarcoma: clinicopathologic analysis of 24 cases. Am J Surg Pathol 31: 1868-1874, 2007.

14. Gaerte SC, Meyer CA, Winer-Muram HT, Tarver RD and Conces DJ: Fat-containing lesions of the chest. Radiographics 22: S61-S78, 2002

15. Attal H, Jensen J and Reyes CV: Myxoid liposarcoma of the anterior mediastinum. Diagnosis by fine needle aspiration biopsy. Acta Cytol 39: 511-513, 1995

16. Barbetakis N, Samanidis G, Paliouras D, Boukovinas I, Kiziridou A and Tsilikas C: A rare cause of mediastinal mass: Primary liposarcoma. J BUON 13: 429-431, 2008.

17. Farah M, Abou-Sleiman P and Bahous J: Primary mediastinal liposarcoma: a case report and review of the literature. J Med Liban 49: 165-169, 2001.

18. Enzinger FM and Weiss SW (eds): Liposarcoma. In: Soft Tissue Tumors. 3rd edition. Mosby, St. Louis, MO, ppD431-D466, 1995.

19. Kara M, Ozkan M, Dizbay Sak S and Kavukçu ST: Successful removal of a giant recurrent mediastinal liposarcoma involving both hemithoraces. Eur J Cardiothorac Surg 20: 647-649, 2001. 